Katarzyna Korzeniowska, Ilona Derkowska, Małgorzata Myśliwiec Klinika Pediatrii, Diabetologii i Endokrynologii GUMed Gdańsk, Poland

\title{
Graves-Basedow disease in adolescent patients with type 1 diabetes mellitus
}

INTRODUCTION: The prevalence of Graves-Basedow disease in adolescents with type 1 diabetes mellitus is around $0.5 \%$. Most often the diagnosis of GD in T1DM patients is made many years after the onset of T1DM. The management of GD can be very difficult and may lead to impaired quality of life.

CASE REPORT: 17-years old male was referred to our Department for reevaluation of Graves-Basedow disease. Patient suffered from T1DM for 14 years, with unsatisfactory metabolic control (HbA1c 9,17\%). Moreover in 2009 he was diagnosed with Hashimoto thyroiditis (elevated thyroid antibodies), however remained euthyroid. In 10.2011 patient was diagnosed with hyperthyroidism and treated with methimazole (MMI) to maintain euthyroidism. Two months before the referral, a trial period off medication had been attempted, but the patient experienced a return of symptoms (fatigue, palpitations, heat intolerance). Family history was significant for autoimmune thyroid disease and T1DM in his mother. On physical examination, the patient had a heart rate of $110 \mathrm{bpm}, \mathrm{BP}$ of $130 / 70 \mathrm{mmHg}$. There was no evidence of ophthalmopathy. Thyroid examination revealed enlarged thyroid gland. Laboratory funds showed recurrence of hyperthyroidism (TSH<0,004uU/ml, fT4 36,76 pmol/I). After discussing both therapeutic options with parents (MMI treatment continuation or radioiodine ablation) the patient was administered I131. Within 2 months of radioiodine therapy, hypothyroidism was achieved, and the patient started thyroid hormone replacement. Three months after definitive therapy, the patient reported improved school performance, decreased fatigue and no abnormalities in heart rhythm. Diabetes metabolic control did not alter during the treatment.

CONCLUSIONS: In reccurent Graves-Basedow hyperthyroidism radioiodine ablation seems to be a good treatment option, highly improving patients' quality of life. 